\title{
Gentamicin-resistant Pseudomonas endophthalmitis after penetrating keratoplasty*
}

\author{
MICHAEL S INSLER,' H DWIGHT CAVANAGH, ' AND LOUIS A WILSON² \\ From the 'Department of Ophthalmology, Tulane School of Medicine, New Orleans, Louisiana, and the \\ 'Department of Ophthalmology, Emory University, Atlanta, Georgia, USA
}

SUMmARY A case of pseudomonas endophthalmitis after penetrating keratoplasty is described. Bacterial sensitivities in this case and in five of six previously reported cases of endophthalmitis after corneal transplant showed resistance to gentamicin, an antibiotic routinely used in the transport medium. The recovery of antibiotic resistant bacteria in antibiotic supplemented media is discussed with emphasis on prevention.

Bacterial endophthalmitis is an uncommon but potentially devastating complication of penetrating keratoplasty. A major source of this kind of infection in corneal transplantation can be the donor material or contaminated medium. We describe a case of pseudomonas endophthalmitis complicating McCarey-Kaufman (M-K) media, its management, and methods for further prevention.

\section{Case history}

On 26 March 1981 a 74-year-old woman had an 8.5 $\mathrm{mm} / 8.0 \mathrm{~mm}$ penetrating keratoplasty with vitrectomy of the left eye for aphakic bullous keratopathy. On the first postoperative day a small central epithelial defect was noted. The anterior chamber was deep, and a small fibrin clot was present. On the second postoperative day a culture was taken and the donor rim was found to have a Gram negative bacillus. Treatment consisting of topical gentamicin and Decadron (dexamethasone) was maintained. Examination early on 29 March 1981, the third postoperative day, showed a hypopyon at the inferior graft interface. The eye was more injected, and the anterior chamber reaction was also more intense. Endophthalmitis was suspected and, because of initial antibiotic sensitivities of the donor rim, the patient was started on ticarcillin $3 \mathrm{~g}$ intravenously

*Presented in part at the Southcast Regional Eyc Bank Mecting, New Orlcans, Louisiana, in Junc 1982.

Correspondence to Dr M S Insler, Department of Ophthalmology Tulane School of Medicinc, 1430 Tulane Avenuc, New Orlcans, Louisiana 70112, USA. every four hours and a pars plana vitrectomy was done. Vitreous cultures were obtained and intravitreal injections of carbenicillin, cephradine, and amikacin were given. Postoperatively the patient was started on prednisone $20 \mathrm{mg}$ orally qid and carbencillin $3 \mathrm{~g}$ intravenously four-hourly. Atropine $1 \%$, Decadron, and fortified gentamicin drops $(14 \mathrm{mg} / \mathrm{ml})$ were all given topically in the left eye. On 30 March 1981 after the vitrectomy there was a marked cellular reaction and a $50 \%$ hypopyon (Fig. 1). Examination on 2 April 1981 showed that the hypopyon had begun to clear. On 7 April 1981 all intravenous antibiotics were stopped, and the patient was discharged on 10 April 1981. Visual acuity on 7 December 1981 was $20 / 300$ with a clear graft.

The donor rim was completed on 31 March 1981 and grew a pure culture of Pseudomonas aeruginosa

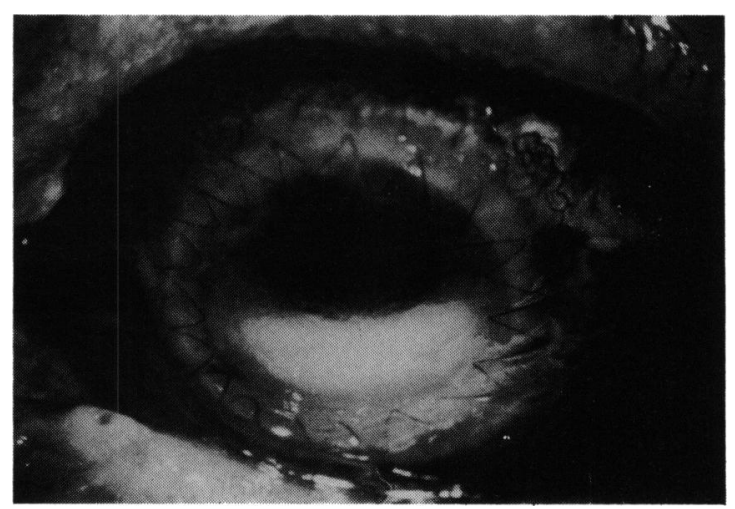

Fig. 1 Corneal transplant with hypopyon after vitrectomy. 
sensitive to carbenicillin, sulphonamide, and amikacin and resistant to ampicillin, chloramphenicol, gentamicin, and cephalothin. Serological typing showed the organism isolated from the donor rim to be identical to the vitreous isolate. A minimum inhibitory concentration (MIC) test was done on the organism to gentamicin, which was greater than 160 $\mu \mathrm{g} / \mathrm{ml}$. A gentamicin assay was obtained from the original corneal transport M-K fluid, which showed a concentration of $49 \mu \mathrm{g} / \mathrm{ml}(\mathrm{mg} / \mathrm{l})$.

\section{Discussion}

LeFrançois and Baum' reported on the first encounter with bacterial endophthalmitis after keratoplasty. The donor cornea was irrigated with Neosporin (neomycin, gramicidin, polymyxin B) drops prior to removing the rim, and gentamicin was added to the $\mathrm{M}-\mathrm{K}$ culture medium to make a final concentration of $6 \mu \mathrm{g} / \mathrm{ml}$. Cultures of the residual donor cornea, the culture media, and from the anterior chamber all grew a 'pseudomonas species' resistant to gentamicin and carbenicillin. The corneal graft eventually developed severe stromal oedema, and the globe slowly became phthisical. The authors believe the following factors may combine to produce a greater incidence of endophthalmitis by a more resistant pathogen with the use of M-K storage. (1) a larger potential inoculum due to $(a)$ increased length of storage, $(b)$ the possibility that tissue culture fluid encourages growth of micro-organisms; (2) contamination of the endothelial surface of the donor cornea; (3) the emergence of more antibiotic resistant micro-organisms in antibiotic supplemented media.

Reports by Shaw, ${ }^{2}$ Khodadoust, ${ }^{3}$ and Escapini ${ }^{4}$ have further substantiated that contamination of donor material can lead to bacterial infection in the host. Cultures obtained from these three cases grew Streptococcus pneumoniae with no antibiotic sensitivites reported; Pseudomonas aerugenosa sensitive only to carbenicillin; and Staphylococcus aureus resistant to chloramphenicol, clindamycin, streptomycin, gentamicin, and penicillin.

More recently Leveille et al. ${ }^{5}$ described four cases of endophthalmitis (three with positive donor rims) in 1876 penetrating keratoplasties. M-K media with gentamicin added at the eye bank was used in all instances. Cultures of the organisms taken from the patient and donor rims showed: case 2, Pseudomonas aeruginosa, resistant to gentamicin and the subject of this report; case 3, group D streptococcus sensitive to gentamicin; and case 4, Streptococcus pneumoniae without antibiotic sensitivities.

Recommendations for the use of M-K medium to improve corneal preservation prior to penetrating keratoplasty includes cold storage between $4^{\circ}$ to $8^{\circ} \mathrm{C}$ and the addition of gentamicin $100 \mu \mathrm{g} / \mathrm{ml}$ to inhibit bacterial growth. Any effort, however, to sterilise donor corneas in transport medium with fortified antibiotics cannot be relied upon with impunity. In fact various clinical case reports, including ours, suggest that antibiotic supplemented $\mathrm{M}-\mathrm{K}$ medium does not sterilise the medium prior to keratoplasty in all instances.

Several authors, indeed, have evaluated the effects of time and temperature on bacterial recovery in M-K medium supplemented with antibiotics. ${ }^{67}$ The results of these studies cast doubt against the expectation that $\mathrm{M}-\mathrm{K}$ medium with antibiotic, as usually used, will reliably kill even sensitive bacteria.

The recovery of gentamicin resistant bacteria (in our case MIC greater than $160 \mu \mathrm{g} / \mathrm{ml}$ and in five of six cases that developed endophthalmitis where antibiotic sensitivities were reported) in gentamicinsupplemented media poses a new problem in the prevention of postoperative infection. No matter what precautions are taken, or what kinds or doses of antibiotic are used prophylactically, some contamination resistant to all these measures is possible. Because of the isolation of gentamicin-resistant organisms we believe storage medium containing gentamicin should be refrigerated at $4^{\circ} \mathrm{C}$ to inhibit the growth of gentamicin-resistant organism. It should then be allowed to reach room temperature one to two hours before corneal transplantation in order for the gentamicin to exert its effect upon gentamicin-sensitive bacteria.

To help further reduce the risk of donor to host transmission of disease we recommend that patients undergoing cornea transplant surgery be treated with parenteral broad spectrum antibiotics, in addition to topical therapy with gentamicin (to cover gentamicinsensitive organisms) until the results of the culture and sensitivities of the donor rim are complete. It has been the policy of one of us (MSI) to give intravenous cephaloridine or piperacillin prophylactically at the time of surgery and to continue such therapy for at least 72 hours postoperatively.

We further emphasise that, although it is not practicable to culture donor corneas once they are immersed in antibiotic supplemented M-K medium, and post-mortem culture results are misleading, it is mandatory to culture the donor rim at the time of surgery.

Finally, an awareness that infection can be transmitted from the donor cornea may also help prevent its occurrence.

\section{References}

1 Le François M, Baum JL. Flavobactcrium endophthalmitis following keratoplasty. Usc of a tissuc culture medium-stored cornca. Arch Ophthalmol 1976; 941: 907-1909. 
2 Shaw EL, Aquavella JV. Pncumococcal cndophthalmitis following grafting of corncal tissuc from a (cadaver) kidncy donor. Ann Ophthalmol 1977; 9: 435-40.

3 Khodadoust AA, Franklin RM. Transfer of bacterial infection by donor cornca in penctrating keratoplasty. Am J Ophthalmol 1979; 87: $130-2$.

4 Escapini H, Olson RJ, Kaufman HE. Donor cornca contamination with McCarcy-Kaufman medium prescrvation. Am J Ophthalmol 1979; 88: 59-62.
5 Leveille AS, McMullan FD, Cavanagh HD. Endophthalmitis following penetrating keratoplasty. Ophthalmology 1983; 90: 38-9.

6 Baum J, Barza M, Kanc A. Efficacy of penicillin G, cefazolin, and gentamicin in M-K medium at $4^{\circ} \mathrm{C}$. Arch Ophthalmol 1978; 96: 1262-4.

7 Graul EE Jr, Olson RJ, Janncy A. Resistance of Pseudomonas aeruginosa to modern cyc-banking techniquc. Ann Ophthalmol $1980 ; 12: 429-32$. 